\title{
Ein integratives soziokognitives Modell des dynamischen Lexikons
}

\begin{abstract}
Dieser Beitrag liefert eine Skizze eines gebrauchsbasierten integrativen soziokognitiven Modells des dynamischen Lexikons. Das Modell besteht aus drei Kernkomponenten: Handlungen in der aktuellen Sprachverwendung, kognitiven Prozessen und sozialen Prozessen. Die Komponenten des Modells werden zunächst einzeln beschrieben und dann zusammengefügt. Es wird gezeigt und anhand von zwei Beispielen illustriert, wie das Modell durch die systematische Beschreibung der Interaktion zwischen diesen Komponenten gleichzeitig Stabilität und Struktur sowie Variation und Wandel im Lexikon vorhersagt.
\end{abstract}

\section{Vorbemerkung}

In diesem Beitrag werden die groben Umrisse eines soziokognitiven Modells der Struktur, Variabilität und Dynamik des Lexikons beschrieben. Die Größe dieses Vorhabens steht in einem eklatanten Missverhältnis zum verfügbaren Umfang des Texts. Dies hat zur Folge, dass die Lektüre des Beitrags einen dreifachen Vertrauensvorschuss erfordert.

Erstens können die zahlreichen Einsichten, die anderen Quellen zu verdanken sind, nur in Form einer pauschalen Danksagung an die folgenden besonders inspirierenden Autoren gewürdigt werden: Peter Auer, Harald Baayen, Karl Bühler, Joan Bybee, Bill Croft, Penelope Eckert, Nick Ellis, Charles Fillmore, Dirk Geeraerts, Adele Goldberg, H.P. Grice, Peter Harder, Rudi Keller, Roman Jakobson, William Labov, Ron Langacker, Elena Lieven, Brian MacWhinney, Herrmann Paul, Ferdinand de Saussure, John Searle, John Sinclair, Len Talmy, Michael Tomasello, Friedrich Ungerer und Alison Wray. Würde ich versuchen, auch nur die wichtigsten Arbeiten dieser Autoren zu nennen, wäre ein Großteil des Beitrags bereits durch die Bibliografie gefüllt.

Zweitens kann ich nur ebenso pauschal versichern, dass zu vielen Fragen, die sich den Lesern dieses Texts unweigerlich aufdrängen werden, bereits Antworten hinterlegt sind. Diese sind entweder in existierenden Veröffentlichungen zu finden (Schmid 2014, 2015, 2016, 2017a, b) oder in aktuell in Vorbereitung befindlichen Texten (Schmid i.Bearb.) schon ausformuliert, können aber hier aus Platzgründen nicht genannt werden. 
Drittens erfordert die Lektüre dieses Beitrags, die Ausführungen in den ersten Abschnitten mit dem Vertrauen zur Kenntnis zu nehmen, dass sich am Ende alles zu einem kohärenten Gesamtbild zusammenfügen wird.

\section{Einleitung: das dynamische Lexikon}

Legen wir zunächst fest, was gemeint sein soll, wenn die Rede vom dynamischen Lexikon ist. Zuallererst ist damit gemeint, dass das Lexikon insgesamt sowie seine Struktur und Elemente dauerhaft Variation und Wandel unterworfen sind. Die Dynamizität lexikalischer Elemente und Strukturen ist ein inhärenter Wesenszug des Lexikons. Von Variation und Wandel sind sowohl die mentalen Lexika einzelner Sprecher betroffen als auch das kollektive Lexikon einer Sprachgemeinschaft. Konkret sind damit u.a. die folgenden bekannten Aspekte angesprochen:

- Neue Lexeme werden geprägt und treten ins Lexikon ein, alte geraten außer Gebrauch, werden archaisch und obsolet.

- Lexeme sind variabel und verändern sich hinsichtlich ihrer phonologischen, graphemischen und morphologischen Formen und ihrer Bedeutungen.

- Lexeme verändern sich hinsichtlich ihres Komplementierungs- und Kollokationsverhaltens.

- Die lexikalischen Oppositionen und Kookkurrenztendenzen und -restriktionen, die einen wesentlichen Beitrag zur Struktur des Lexikons leisten, sind variabel und verändern sich.

- Lexeme verlieren und erobern typische Benutzungskontexte und verändern ihre Konnotationen und soziale Indexikalität, d.h. ihr Potenzial, Auskunft über soziale und individuelle Merkmale ihrer typischen Sprachbenutzergruppen und Sprachbenutzer zu geben.

Das vorgeschlagene Modell des dynamischen Lexikons zielt darauf ab, diese Aspekte abzubilden. Aus Gründen, die noch ersichtlich werden, bezeichne ich das Modell als „Entrenchment- und Konventionalisierungsmodell“ (Schmid 2015). Im Zentrum der Betrachtungen stehen die folgenden Fragen:

1. Warum ist das Lexikon dynamisch?

2. Kann man das „Warum“ und das „Wie“ modellieren?

3. Kann man die Prinzipien und Pfade der Dynamik des Lexikons systematisch „,vorhersagen“?

Zur Beantwortung dieser Fragen werden zunächst Annahmen darüber dargelegt, wie lexikalisches Wissen in den Köpfen einzelner Sprecher verarbeitet wird und 
repräsentiert ist (Abschnitt 3) und wie es zu einer Übereinkunft über das kommunikative Potenzial von Wörtern in einer Sprachgemeinschaft oder Teilen derselben kommt (Abschnitt 4). Auf der Basis dessen wird in Abschnitt 5 ein dynamisches Modell des Lexikons entwickelt, das in den darauffolgenden Abschnitten im Hinblick auf seine Vorhersagen zur Struktur und Stabilität (Abschnitt 6) und zur Variabilität und Dynamik (Abschnitt 7) geprüft wird. Abschnitt 8 liefert Illustrationen von zwei typischen Szenarien lexikalischer Dynamik.

\section{Kognition: Was bedeutet es, ein Wort zu kennen?}

Der hier vorgestellte Ansatz geht von der Annahme aus, dass Wissen über Wörter bzw. Lexeme in Form der folgenden vier Typen von Assoziationen repräsentiert ist:

1. Symbolische Assoziationen zwischen der Form bzw. den Formen des Lexems und der Bedeutung bzw. den Bedeutungen.

2. Paradigmatische Assoziationen zwischen einer lexikalischen Form oder Bedeutung und anderen Formen und Bedeutungen, die während der Sprachverarbeitung im assoziativen Netzwerk als Mitbewerber aktiviert werden und als solche repräsentiert sind.

3. Syntagmatische Assoziationen zwischen sequenziell angeordneten sprachlichen Formen und ihren Bedeutungen.

4. Pragmatische Assoziationen zwischen dem Gebrauch von Lexemen und den situativen Gebrauchsumständen, also v.a. Sprachbenutzern und ihrer sozialen Merkmale und Beziehungen, Ort und Zeit, Objekten usw.

Diese vier Typen von Assoziationen, und nichts außer ihnen, sind sowohl für die aktuelle kognitive Verarbeitung im Sprachgebrauch als auch für die Repräsentation von Wissen über lexikalische Einheiten verantwortlich. Im Langzeitgedächtnis repräsentiertes lexikalisches Wissen kommt durch die Verfestigung - das Entrenchment - der während der Verarbeitung aktivierten Assoziationsmuster zustande und verändert sich permanent unter dem Einfluss des Gebrauchs. Der kognitive Prozess, der für die Verfestigung sorgt, ist die Routinisierung, d.h. die Stärkung von Assoziationsmustern durch ihre wiederholte Aktivierung. Diese beruht auf der Ähnlichkeit wiederkehrender kommunikativer Anforderungen in Sprachproduktion und -rezeption, also auf der wiederholten Verarbeitung als identisch behandelter Äußerungsteile oder Gemeinsamkeiten derselben. In Abhängigkeit vom Ausmaß der Ähnlichkeit dessen, was routinisiert wird, beinhaltet dieser Prozess eine mehr oder weniger stark ausgeprägte Schematisierung. Bei 
hoher Ähnlichkeit, z.B. bei der Routinisierung der Assoziationsmuster für einzelne unregelmäßige Wortformen wie $a \beta$, trank oder schlief ist die Schematisierung trotz der Variabilität der verschiedenen Gebrauchsereignisse (z.B. hinsichtlich ihrer phonetischen Realisierung, Kotexte, Kontexte und aktuellen Bedeutungen) kaum bemerkbar. Bei geringer Ähnlichkeit, etwa wenn verschiedene Formen wie isst, $a \beta$, essen und gegessen als Manifestationen des Lexems ESSEN gespeichert werden, ist der Anteil der Schematisierung an der Routinisierung prominenter.

Aus lerntheoretischer Sicht lässt sich der Prozess der Routinisierung als eine Form impliziten, statistischen Lernens begreifen, das über die vier Typen von Assoziationen operiert. Bedeutungen werden auf der Grundlage sprachlicher Erfahrung probabilistisch mit Formen assoziiert; Situationen werden probabilistisch mit Bedeutungen und Formen assoziiert, die in vergleichbaren Situationen angetroffen wurden; Kookkurrenztendenzen werden ebenfalls basierend auf sprachlicher Erfahrung als probabilistische syntagmatische Assoziationen, und Konkurrenzverhältnisse als paradigmatische Assoziationen gelernt. Gedächtnistheoretisch findet einerseits die Konsolidierung von Assoziationsmustern vom episodischen zum semantischen Gedächtnis statt, andererseits aber auch eine zunehmende Prozeduralisierung der sequenziellen Verarbeitung (vgl. Schmid 2017b).

Mit den kognitiven Prozessen der Assoziation, Routinisierung und Schematisierung sind die Kernelemente einer gebrauchsbasierten, kognitiven Konzeption des individuellen mentalen Lexikons skizziert. Gemäß dieser Konzeption sind der produktive und rezeptive Sprachgebrauch Voraussetzungen sowohl für die Aktivierung als auch die Repräsentation von Assoziationsmustern. Ob und wann dieser Gebrauch stattfindet, liegt - wenn man vom inneren Monolog oder dem Selbstgespräch absieht - nicht mehr allein in der Macht des einzelnen Sprachbenutzers, sondern in der Sprachgemeinschaft und den Strukturen und Prozessen, die sie kennzeichnen. Diesen wenden wir uns nun zu.

\section{Gesellschaft: Wie stellt die Sprachgemeinschaft eine Übereinkunft her?}

Ein Wort zu kennen, heißt nicht nur, ein bestimmtes Assoziationsmuster im assoziativen Netzwerk aktivieren zu können. Vielmehr beinhaltet die Kenntnis eines Wortes die Teilhabe an einer Konvention, d.h. an der in einer Sprachgemeinschaft vorhandenen Übereinkunft darüber, welche Formen das Wort hat und wie und unter welchen Bedingungen es mit bestimmten kommunikativen Wirkungen verwendet werden kann. Konventionen sind in Sprachgemeinschaften nicht nur dis- 
tribuiert, sondern müssen stillschweigend gegenseitig anerkannt sein. Damit sind sie nicht mehr allein kognitiv repräsentiert, sondern haben eine inhärente soziale Komponente. Es gilt nun, die soziokognitiven und sozialen Prozesse zu beleuchten, die dazu führen, dass die Mitglieder einer Sprachgemeinschaft die Intuition entwickeln, dass sie gegenseitig Wissen über lexikalische Einheiten teilen und anerkennen. Aus Sicht des hier vorgestellten Modells, sind die folgenden fünf Prozesse für die Konventionalisierung lexikalischer Einheiten erforderlich bzw. ihr zuträglich.

Der erste Prozess ist die Ko-Semiose, d.h. die gemeinsame und gegenseitige Aushandlung der Bedeutung in einer Sprachgebrauchssituation. Wie bereits angedeutet, reicht es für erfolgreiches Verstehen nicht aus, wenn die an einer Sprachgebrauchssituation beteiligten Sprachbenutzer lediglich jeweils einzeln ausgelöst durch den Gebrauch von Wörtern mentale Repräsentationen aktivieren. Vielmehr müssen sie gleichzeitig unter dem Eindruck stehen, dass sie das Verständnis dieser Wörter gegenseitig teilen, dergestalt, dass ein Teilnehmer A zu wissen glaubt, was der andere Teilnehmer B denkt, und gleichzeitig zu wissen glaubt, dass der andere Teilnehmer B weiß, dass A dasselbe denkt und ebenfalls weiß, dass A weiß, dass B das weiß. Dieser Prozess hat neben der kognitiven eine genuin interpersonale Komponente, da er zwischen verschiedenen Menschen und nicht nur innerhalb einzelner abläuft. Werden konventionalisierte Lexeme nicht durch ständige Ko-Semiose aufgefrischt, so drohen sie außer Gebrauch zu geraten und obsolet zu werden. Ist dieser Aushandlungsprozess der Ko-Semiose bei einem Gebrauch eines neuen Lexems nicht ausreichend erfolgreich, zum Beispiel weil Beteiligte nur eine diffuse Vorstellung der Bedeutung des Wortes aktivieren können und nicht den Eindruck gewinnen, dass sie an der Ko-Semiose in vollem Ausmaß partizipieren, dann ist der Weg in Richtung einer späteren Konventionalisierung und Aufnahme ins Lexikon bereits verbaut.

Ko-Semiose kann, muss aber nicht zwingend, zur Ko-Adaption führen. Mit diesem Prozess ist die Tendenz angesprochen, Aspekte zuvor verarbeiteter Äußerungen aufzunehmen. Bekannt unter Bezeichnungen wie Akkommodation, Alignment oder Priming, können solche Wiederaufnahmen alle sprachlichen Ebenen, von der Aussprache einzelner Segmente über Betonungsmuster, Satzstrukturen und nicht zuletzt auch lexikalische Einheiten betreffen. Mit der Wahl des Terminus Ko-Adaption soll das dynamische und interaktive Element dieses Prozesses hervorgehoben werden. Bezogen auf das Lexikon ist damit die Wiederholung einer lexikalischen Einheit durch einen Hörer in einem späteren Redebeitrag innerhalb derselben Sprachgebrauchssituation gemeint. Ko-Adaption ist keine notwendige Bedingung für spätere Konventionalisierung; sie dürfte Letzterer aber zuträglich sein, weil die aktive Verwendung die aktivierten Assoziationsmuster festigt, was die Wahrscheinlichkeit erhöht, dass Gedächtnisspuren in andere Gesprächssi- 
tuationen hinüber gerettet werden. Dies wiederum ist eine zwingende Voraussetzung dafür, dass ein neu geprägtes Wort in einer Sprachgemeinschaft konventionalisiert werden kann.

Kernelement dieser Konventionalisierung ist die Bereitschaft der Mitglieder der Sprachgemeinschaft, ein bestimmtes Wort oder eine lexikalische Fügung als Mittel zur Lösung einer kommunikativen Aufgabe zu verwenden. Das Zustandekommen dieser Bereitschaft lässt sich als Usualisierung begreifen. In diesem Prozess ist der Kern dessen angelegt, was als Konvention bezeichnet wird, nämlich die stillschweigende gegenseitige Übereinkunft über das kommunikative Potenzial eines Lexems oder Ausdrucks. Ursprung der Usualisierung ist die Ko-Semiose in einer konkreten Gebrauchssituation. Für die breitere Usualisierung über diese hinaus ist es aber erforderlich, dass Wörter auch in anderen Situationen Verwendung finden.

Während Usualierung auf die Übereinkunft hinsichtlich des kommunikativen Nutzens und Potenzials an sich bezogen ist, betrifft die Diffusion die Verbreitung in sozialen Gruppen und communities of practice sowie die Pfade, auf denen diese Verteilung in Abhängigkeit von sozialen Strukturen und Prozessen abläuft. Usualisierung und Diffusion müssen auseinandergehalten werden, weil die beiden Prozesse nicht kollinear sind. Fachterminologie kann als Beispiel für den Fall dienen, dass ein hoher Grad an Usualisierung in einem begrenzten Teil der Sprachgemeinschaft zu beobachten ist. Unüblicher Amtswortschatz liefert das komplementäre Beispiel, bei dem ein hoher Grad an Diffusion mit geringer Bereitschaft, das entsprechende Vokabular als Lösung für kommunikative Aufgaben zu verwenden, gepaart ist. In Korpusstudien kann der Grad der Usualisierung durch die relative Häufigkeit von Wörtern (am besten im Vergleich $\mathrm{zu}$ onomasiologischen Alternativen) approximiert werden; als Maß der Diffusion wäre dagegen zusätzlich die Dispersion über Sprachbenutzer, soziale Gruppen und Verwendungskontexte heranzuziehen.

Als letzte Teilprozesse beinhaltet die Konventionalisierung die implizite und explizite Normierung. Usualierung und Diffusion führen zu normativem Druck, Wörter so zu verwenden, wie es die anderen Mitglieder der Sprachgemeinschaft oder Gruppe tun. In dokumentierten Sprachen mündet dieser implizite Normierungsprozess in einen expliziten, d.h. in die Kodifizierung der Konvention in Lexika, Grammatiken oder Handreichungen zum gelungenen Gebrauch von Wörtern und Ausdrücken. 


\section{Interaktion zwischen Kognition und Gesellschaft: Gebrauch, Entrenchment und Konventionalisierung}

Bisher ist behandelt worden, wie lexikalisches Wissen in den Köpfen einzelner Sprecher verarbeitet wird und in unterschiedlicher Tiefe repräsentiert ist, und wie Konventionen über das kommunikative Potenzial von Wörtern zustande kommen und aufrecht erhalten werden. Beide Aspekte wurden als inhärent dynamisch modelliert: Assoziation, Routinisierung und Schematisierung sind ständig ablaufende kognitive Entrenchmentprozesse; Ko-Semiose, Ko-Adaption, Usualisierung, Diffusion und Normierung sind soziale Konventionalisierungsprozesse. Für das Verständnis des dynamischen Lexikons ist es nun erforderlich zu begreifen, wie diese Prozesse miteinander interagieren.

Zwingende Voraussetzung für das Zusammenwirken der kognitiven und sozialen Prozesse ist die Verwendung lexikalischer Einheiten in konkreten Gebrauchssituationen in mündlichen, schriftlichen oder anderen Medien (Zeichensprache, elektronisch übermittelte Sprache). Produktive und rezeptive kommunikative Handlungen in situierten Gebrauchsereignissen stehen deshalb im Zentrum des Modells (vgl. Abb. 1, die die Interaktion zwischen kognitiven und sozialen Prozessen schematisch darstellt und als Anker für die folgenden Ausführungen dienen wird).

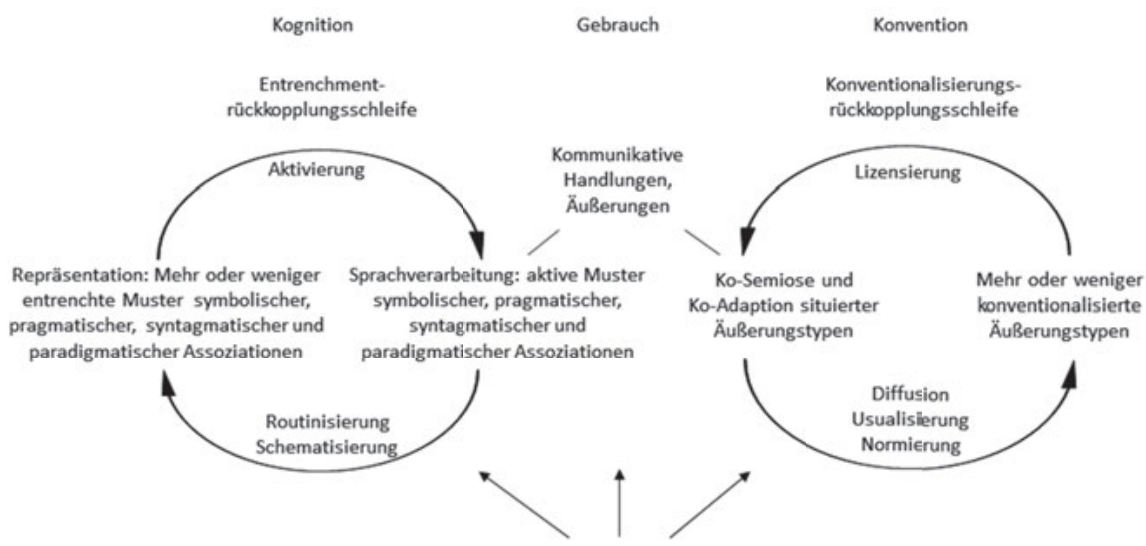

Kognitive, emotiv-affektive, pragmatische und soziale Kräfte

Abb. 1: Grafische Darstellung des Entrenchment- und Konventionalisierungsmodells 
Kommunikative Handlungen stellen das Rohmaterial für Entrenchment- und Konventionalisierungsprozesse dar. Beide Prozesse sind als selbstreferenzielle Rückkopplungsschleifen zu verstehen. Im kognitiven System werden Äußerungen in Form von Mustern von symbolischen, pragmatischen, syntagmatischen und paradigmatischen Assoziationen verarbeitet. Wiederholt sich die Verarbeitung, weil sich sprachliche Elemente und/oder zu enkodierende Inhalte wiederholen, werden diese Muster routinisiert und dabei je nach Ausmaß der Gemeinsamkeit, dessen, was routinisiert wird, schematisiert. Je nach Frequenz dieser Wiederholungen werden entsprechende Muster mehr oder weniger verfestigt und sind mehr oder weniger stark repräsentiert. In Abhängigkeit von ihrer Gebrauchshäufigkeit (und anderen Faktoren) haben Lexeme im assoziativen Netz insofern eine privilegierte Stellung, als sie als Attraktoren dienen, die Aktivierung leichter und schneller anziehen als lexikalisch nicht-verfasste Konzepte oder selten aktivierte Syntagmen. Verfestigung resultiert damit in einer zunehmenden Automatisierung der Aktivierung und Verarbeitung. Diese Annahmen sind in Einklang mit psycholinguistischen Kernbefunden zur Verarbeitung und Repräsentation lexikalischer Einheiten, u.a. mit dem Wortüberlegenheitseffekt, dem Häufigkeitseffekt und Kontexteffekten.

Die Aktivierungsneigung von Assoziationsmustern korreliert mit dem Grad der Verfestigung: Häufig Verarbeitetes wird leichter wieder aktiviert als selten oder noch nie Verarbeitetes. Damit schließt sich die Entrenchmentrückkopplungsschleife, denn häufig Aktiviertes erfährt stärkeres Entrenchment, was sich wiederum in schnellerer und leichterer Aktivierung niederschlägt.

Die Rückkopplungsschleife der Konventionalisierung verläuft insofern grundsätzlich analog dazu, als Frequenz der Wiederholung eine wesentliche Antriebskraft für Konventionalisierung darstellt. Was bereits konventionalisiert ist, wird eher wieder verwendet als Originelles, u.a. weil gegenseitiges Verstehen durch die Verwendung voll lizenzierter Äußerungstypen wahrscheinlicher ist. Wie in Abbildung 1 angezeigt, operieren die fünf in Abschnitt 4 erläuterten Prozesse nicht über Assoziationen, sondern über situierte Äußerungstypen, d.h. sprachliche Oberflächenphänomene. Nicht die mentalen Repräsentationen werden konventionalisiert, sondern die Übereinkunft über die Formen und kommunikativen Wirkungen von Lexemen und Ausdrücken.

Entrenchment und Konventionalisierung interagieren nur durch den Gebrauch in konkreten sozialen Kontexten miteinander. Als Vermittler zwischen Kognition und Konvention spielen dabei pragmatische Assoziationen, die Information aus Gebrauchssituation, auch solche über soziale Eigenschaften und Beziehungen, in den Entrenchmentzyklus einspeisen, sowie der soziokognitive Prozess der Ko-Semiose eine zentrale Rolle. (Die Darstellung der beiden Rückkopplungsschleifen in Abbildung 1 ist übrigens insofern irreführend, als sich die 
beiden Kreise im Gebrauch überlappen sollten. Dies würde aber die Lesbarkeit der Abbildung noch weiter beeinträchtigen.)

Entrenchment- und Konventionalisierungsprozesse sowie die Interaktion zwischen ihnen unterliegen der Wirkung verschiedener Typen externer Kräfte, die in Abbildung 1 nur angedeutet sind. Diese kommen im Folgenden in Ansätzen zur Sprache.

\section{Struktur: stabilisierende Momente}

Wie generiert das in Abschnitt 5 skizzierte dynamische System lexikalische Struktur, und wie hält sie diese zumindest scheinbar aufrecht?

Seine grundlegende Struktur erhält das Lexikon von der Seite der kognitiven Prozesse, genauer durch die Routinisierung von und Kooperation und Kompetition zwischen den vier Typen von Assoziationen. Oppositionen, ein wesentliches Strukturprinzip, sind in Form von gefestigten paradigmatischen Assoziationen verfügbar, Kookkurrenzrestriktionen und -tendenzen in Form von mehr oder weniger gefestigten syntagmatischen Assoziationen. Paradigmatische Assoziationen fungieren als kognitives Substrat sprachlicher Phänomene wie Flexionsparadigmen, Sinnrelationen und Wortfelder. Syntagmatische Assoziationen sind maßgeblich für die Verarbeitung und Repräsentation u.a. von Wortbildungsmustern, halbfesten und festen Fügungen und idiomatischen Ausdrücken sowie formelhafter Sprache generell. Wissen über Konnotationen, Stilebenen und soziale Bedeutungen von Formen und Bedeutungen wird durch die Verfestigung pragmatischer Assoziationen gewonnen und repräsentiert. Pragmatische Assoziationen zwischen Gebrauchskontexten und lexikalischen Formen und Bedeutungen sind letztlich auch der Ursprung für die Aktivierung und Verfestigung von symbolischen Assoziationen, indem Wörter mit Aspekten der Gebrauchssituation assoziiert werden.

Dieses kognitiv motivierte System allein ergibt aber natürlich keine Sprache bzw. kein Lexikon, so lange es nicht ständig durch den soziokognitiven Prozess der Ko-Semiose, vermittelt durch pragmatische Assoziationen gestützt und aufgefrischt wird. Findet Ko-Semiose statt, so ist gleichzeitig ein Ausgangs- und beständiger Erneuerungspunkt zur Bewahrung konventionalisierter Äußerungstypen gegeben.

Daraus lässt sich die folgende Konstellation stabilisierender Momente im Modell ableiten: Grundsätzlich sind alle Gebrauchshandlungen, Entrenchmentprozesse und Konventionalisierungsprozesse stabilisierend, solange sie auf dem Prinzip der Wiederholung beruhen. Die Wiederholung von lexikalischen Einheiten und Ausdrücken unter denselben oder ähnlichen Bedingungen im Sprachge- 
brauch trägt zur weiteren Festigung bereits gefestigter Assoziationsmuster und zur weiteren Konventionalisierung bereits konventioneller Äußerungstypen bei. Sprecher, die mit einer wiederkehrenden kommunikativen Aufgabe konfrontiert sind, neigen v.a. in ungezwungenen Kontexten im mündlichen Medium zur Wiederholung. In der Gesellschaft trägt die wiederholte Verwendung zur Aufrechterhaltung einer Konvention bei, indem sie anzeigt, dass ein Wort oder Ausdruck weiterhin als angemessenes Mittel zur Bewältigung dieser kommunikativen Aufgabe angesehen wird.

Die Stabilität der vorhandenen Assoziationen und Mittel wird durch eine Reihe von externen Kräften unterstützt, die Wiederholung fördern. Als wesentliche kognitive Kraft fungiert das Ökonomieprinzip: Sage das, was du unter vergleichbaren Umständen immer sagst. Eine wichtige stabilisierende pragmatische Kraft ist die kommunikative Effizienz: Sage das, was in vergleichbaren Situation kommunikativ schon früher erfolgreich war; arbeite mit deinem Kommunikationspartner aktiv an der Ko-Semiose, d.h. etabliere einen common ground, bemühe dich um audience design. Solidarität zwischen den Interaktanten sowie gemeinsame Zugehörigkeit zu einer sozialen Gruppe sind der Wiederholung von Gefestigtem und Konventionalisiertem ebenfalls zuträglich.

\section{Variation und Wandel: dynamisierende Momente}

Ein kurzer kritischer Blick auf das in Abschnitt 6 skizzierte Szenario entlarvt dieses als simplistisch. Ein erster wichtiger Grund für sein Scheitern liegt in der irrigen Annahme, dass sich Sprachgebrauchsereignisse mehr oder weniger identisch wiederholen. Wäre dies der Fall und käme es häufig vor, so hätte das Szenario der stabilisierenden Momente tatsächlich eine beträchtliche Vorhersagekraft, und Sprachen wären generell stabiler. Tatsache ist aber, dass sich die Anforderungen an Gesprächsereignisse permanent ändern. Sprecher können sich schon allein deshalb nicht dauerhaft innerhalb ihres gewohnten Entrenchmentzyklus bewegen, weil sie sich mit ständig variierenden situationalen, kognitiven und sozialen Anforderungen konfrontiert sehen. Themen von Gesprächen und schriftlichen Texten, angesprochene bzw. intendierte Leserschaft, situationale und soziale Angemessenheit - all dies sind Momente im Sprachgebrauch selbst, die das Verharren in verkrusteten Gewohnheiten erschweren. Hinzu kommt, dass Sprachbenutzer ständig mit der Rezeption des Outputs anderer Sprachbenutzer zu tun haben, die ihrerseits aufgrund anderer sozialer oder individueller Faktoren andere entrenchmentbedingte Gewohnheiten und Präferenzen entwickelt haben. Diese mögen zwar genauso wie die eigenen durch konventionalisierte Äußerungstypen lizen- 
ziert sein, müssen aber trotzdem nicht dem entsprechen, was der Hörer in derselben Situation gesagt oder geschrieben hätte. Auswirkungen des ,Neuen` auf den Entrenchmentzyklus des Hörers sind vorprogrammiert; wie stark sie sich mittelund langfristig als Lernprozess auswirken, hängt von zahlreichen kognitiven, sozialen und emotiv-affektiven Variablen ab.

Dynamisierende Kräfte sind neben dem Gebrauch auch in allen anderen Komponenten des Modells angelegt.

Im Entrenchmentzyklus entfalten die Kooperation und v.a. der Wettbewerb zwischen den verschiedenen Assoziationstypen um Verfestigung eine sehr große dynamisierende Wirkung. Ein Beispiel hierfür wird in Abschnitt 8.2 etwas ausführlicher diskutiert. Es wird zeigen, dass die wiederholungsbedingte Verfestigung syntagmatischer Assoziationen systematische Auswirkungen auf symbolische, paradigmatische und pragmatische Assoziationen hat. Dies bewirkt Prozesse, die in der Sprachwandelforschung als Lexikalisierung und Grammatikalisierung bekannt sind. Die Verfestigung paradigmatischer Assoziationen trägt zum analogischen Wandel bei, und zwar dadurch, dass systematische Oppositionen z.B. in der Flexionsmorphologie auf Bereiche durchschlagen, die zuvor davon nicht betroffen waren. Dass häufige unregelmäßige Formen davon bekanntlich nicht betroffen sind, lässt sich durch die aufgrund höherer Verwendungsfrequenz größere Stärke der symbolischen Assoziation zwischen den unregelmäßigen Formen und ihren Bedeutungen erklären. Die Verfestigung pragmatischer Assoziationen kann zu kontextinduziertem Wandel führen. Hierbei werden zuvor kontextabhängige pragmatische Assoziationen durch zunehmende Wiederholung allmählich semantisiert, d.h. als symbolische Assoziationen unabhängig vom aktuellen Kontext routinisiert.

Hauptursachen für Variation und Wandel durch Konventionalisierungsprozesse sind die Ko-Semiose und Ko-Adaption zwischen Mitgliedern unterschiedlicher sozialer Gruppen. Je nach Ausmaß der sozialen Distanz ist mit verschieden großen sozial bedingten Unterschieden in den Sprachbiografien zu rechnen, die sich in jeweils verschiedenen Entrenchmentzuständen niederschlagen. Finden die genannten Prozesse zwischen Mitgliedern stark verschiedener sozialer Gruppen statt, so ist der mögliche Beitrag zur Diffusion besonders groß, gleichzeitig ist aber auch die Gefahr besonders hoch, dass Ko-Semiose scheitert und ein Missverständnis als Ausgangspunkt für Sprachwandel fungiert. Extreme Fälle des Aufeinandertreffens verschiedener sozialer Gruppen sind Sprachkontaktsituationen, die die Möglichkeit in sich bergen, Lehnwörter, die per definitionem bisher weder assoziativ verfestigt noch konventionalisiert sind, per Ko-Semiose und Ko-Adaption in die Entrenchmentzyklus der an einer Gesprächssituation Beteiligten und den Konventionalisierungszyklus der Sprachgemeinschaft einzuspeisen. Ein weiterer Motor dynamisierender Momente in den Konventionalisierungsprozessen ist die Normie- 
rung selbst, die - wie vom Konzept der Rückkopplungsschleife vorhergesagt sowohl als Prozess wie auch als Kraft wirkt. Implizite und explizite Normierung veranlassen Sprachproduzenten dazu, sich situationsgemäß an Normen z.B. der gehobenen oder der geschriebenen Sprache zu orientieren, was sie dazu zwingen kann, Gewohnheiten zu überwinden, und damit auch die Gefahr erhöht, Malapropismen, Hyperkorrektes und andere Ungeschicklichkeiten zu produzieren.

Schließlich können die externen Kräfte dynamisierende Wirkung entfalten. Im Bereich der externen kognitive Kräfte ist hier v.a. die Aktivierungsverbreitung im assoziativen Netzwerk zu nennen, die zu Innovationen wie metaphorischen oder metonymischen Übertragungen oder auch taxonomischem Wandel führen kann. Dies wird häufig begünstigt durch pragmatische, soziale und emotiv-affektive Kräfte wie Extravaganz, soziale Ambitionen, Prestige oder das Bemühen, kreativ und originell zu sein, um Gesprächspartner zu beeindrucken. All dies kann Sprecher dazu veranlassen, ihr gewohntes und ,entrenchtes“ sowie konventionalisiertes Terrain bewusst oder unbewusst zu verlassen, was sich letztendlich, wie von dynamisch-adaptiven Systemtheorien vorhergesagt, als kleiner Auslöser für eine große und langfristige Veränderung im kognitiven und im sozialen System erweisen kann.

\section{Exemplarische Anwendungen: zwei typische Muster lexikalischer Dynamik}

Um das Potenzial des skizzierten Modells aufzuzeigen, werden im Folgenden exemplarisch zwei Konstellationen von Gebrauchshandlungen, Prozessen und Kräften erläutert, die unterschiedliche Formen lexikalischer Dynamik hervorbringen. Zunächst geht es um lexikalische Innovation (8.1) und dann um die Idiomatisierung usueller Wortverbindungen (8.2).

\subsection{Lexikalische Innovation aus Sicht des Modells}

Als konkretes Beispiel für eine lexikalische Innovation verwende ich das beliebig ausgewählte Substantiv Swag, das vom Langenscheidt-Verlag auf der Basis von konvergierenden Internet- und Juryvoten zum Jugendwort des Jahres 2011 erklärt wurde. Laut Pressemitteilung vom 5.12.2011 steht das Wort „für eine ,beneidenswerte, lässig-coole Ausstrahlung‘ sowie eine ,charismatisch-positive Aura““. Der Ursprung des Wortes liegt im US-amerikanischen Hip-Hop, wahrscheinlich in dem Song Turn my swag on des Rappers Soulja Boy. 
Als Lehnwort gelangt das Wort Swag über den Prozess der Ko-Adaption ins Deutsche. Eine alternative Möglichkeit, die hier aber eben gerade nicht vorliegt, wäre die Schöpfung eines neuen Wortes mit den Mitteln der Wortbildung gewesen. In diesem Fall wäre der Ursprung nicht im Konventionalisierungszyklus, sondern im Entrenchmentzyklus des Schöpfers oder der Schöpferin zu verorten gewesen, der oder die mithilfe von Assoziationen, die bereits im Netzwerk angelegt sind, eine neue Form kreiert. Der soziale Nistplatz der erstmaligen Ko-Adaption liegt im Milieu der Jugendkultur, deren konventionalisierter Sprachgebrauch unter anderem von sozialen Kräften wie dem Interesse an und der Sympathie für die amerikanische Hip-Hop Musik und Szene als identitätsbildendem Moment, covert prestige sowie Distanzierung vom Mainstream beeinflusst wird. Die Neigung zur Dynamik des Lexikons generell und die Übertragung aus dem Englischen ganz konkret werden von diesen sozialen Kräften erheblich begünstigt. Die erstmalige Ko-Adaption und spätere Diffusion des Wortes wurde darüber hinaus durch die Prominenz des Schöpfers im Englischen und die Verbreitung durch den erfolgreichen Song - sowie eine deutsche Coverversion unter dem Titel Dreh den Swag auf - gefördert. Die Tatsache, dass das englische Wort Swag in der einschlägigen Bedeutung bis heute (20.10.2017) nicht in der ständig aktualisierten Online-Version des „Oxford English Dictionary“ verzeichnet ist, spricht dafür, dass Usualierung und vor allem auch Diffusion in breite Schichten der englischen Sprachgemeinschaft hinein trotzdem noch begrenzt sind. Dasselbe dürfte für das deutsche Lehnwort gelten, das noch nicht im Duden eingetragen ist. Mir war das Wort bis vor Kurzem auch nicht bekannt, was sich im Rahmen des Modells durch meine fehlende Teilhabe an einschlägigen sozialen Gruppen erklären lässt. Dass ich das Wort auch von meinen aktuell im Jugendalter befindlichen Kindern nicht zu hören bekommen hatte, bevor ich auf anderem Weg darauf aufmerksam wurde, ist mit seiner sozialen Bedeutung als in-group-Marker zu erklären, der es Jugendlichen eher verbietet, das Wort im Diskurs mit Erwachsenen zu verwenden. Diese soziale Bedeutung und Funktion ist durch den öffentlichen Normierungsprozess im Rahmen der Ernennung zum Jugendwort des Jahres 2011 beschädigt worden, was vermutlich mit dazu beigetragen hat, dass das Wort nach Angabe zahlreicher Jugendlicher, die ich befragt habe, auch unter ihnen an Usualität eingebüßt hat.

Aus Sicht des Entrenchments ist bemerkenswert, dass für Außenseiter wie mich eine Routinisierung von Assoziationen trotz des metalinguistischen Diskurses, an dem ich mich auch hiermit beteilige, kaum stattgefunden hat. Das Wort ist mir jetzt bekannt, aber nicht vertraut. Es ruft in mir nach wie vor keine starke symbolische Assoziation hervor. Entsprechend diffus bleibt die Bedeutung. Syntagmatische Assoziationen, die repräsentieren, wie das Wort im Kotext verwendet wird, sind ebenfalls kaum routinisiert, was zu Fehlverwendungen wie ${ }^{\star}$ Der ist 
ein Swag anstelle von korrekt Der hat Swag führt. Auch über gefestigte paradigmatische Assoziationen, die repräsentieren könnten, mit welchen anderen Substantiven Swag in Relationen wie Synonymie, Hyponymie oder Antonymie steht, verfüge ich im Gegensatz zu meinen Kindern nicht. Und schließlich ist für mich die einzige Situation, die per pragmatischer Assoziation den Gebrauch des Wortes aktiv hervorruft, bisher die des metalinguistischen Diskurses.

Die Spezifika der für das Wort Swag skizzierten Konstellation von Kräften und Konventionalisierungs- und Entrenchmentprozessen kommen eigentlich erst dann richtig zur Geltung, wenn man diesem Beispiel andere Fälle gegenüberstellt, v.a. solche von schneller und hochgradiger Konventionalisierung und tiefem Entrenchment wie z.B. das Lehnwort Smartphone oder das heimische Wortbildungsprodukt Rettungsschirm.

\subsection{Festigung und Idiomatisierung usueller Wortverbindungen}

In diesem Abschnitt geht es um die Frage, wie das Modell den Festigungsprozess usueller Wortverbindungen und den häufig damit einhergehenden Teilverlust der Kompositionalität erklärt. Als Beispiel dient die Wortverbindung aus gegebenem Anlass. Laut den Referenz- und Zeitungskorpora des Digitalen Wörterbuchs der Deutschen Sprache (DWDS) ist diese Sequenz zum ersten Mal im Jahr 1946 belegt und verzeichnet dann nach sporadischen Belegen in den 1950ern einen kontinuierlichen Häufigkeitsanstieg von 0,28 Belegen pro Million Wörter in den 1960ern bis auf 0,43 im ersten Jahrzehnt dieses Jahrhunderts. Im Zuge dieser Frequenzzunahme ist auch eine Veränderung in Richtung einer satzinitialen Routineformel zu beobachten, die einen Aufruf einleitet, einen Missstand zu beheben. Dass diese Funktion und Bedeutung als usuell bzw. usualisiert anzusehen ist, zeigt sich auch darin, dass die Sequenz in quasi zitierenden Anspielungen verwendet wird und Gegenstand metalinguistischer Normierungsprozesse geworden ist. Der Webseite stil.de kann beispielsweise entnommen werden, dass der „Große Knigge“ dazu rät, den Ausdruck in typischen Kontexten - z.B. wenn dazu aufgerufen wird, die Teeküche im Büro sauber zu halten - durch „Wir erinnern daran:“ zu ersetzen.

Den im Modell angelegten kognitiven Erklärungsmechanismus für die Entwicklung dieses Beispiels und vergleichbarer Fälle bezeichne ich als Prinzip der syntagmatischen Verfestigung. Dieses Prinzip besteht aus zwei Komponenten:

1. Die Wiederholung identischer Wortsequenzen resultiert in der Stärkung syntagmatischer Assoziationen zwischen den Elementen, die durch pragmatische Assoziationen unterstützt werden kann. 
2. Wenn dies geschieht, d.h. wenn syntagmatische Assoziationen zwischen den Teilen einer rekurrenten Sequenz durch Wiederholung gefestigt werden, werden

- symbolische, paradigmatische und pragmatische Assoziationen der Teile schwächer,

- während symbolische, paradigmatische und pragmatische Assoziationen des Ganzen stärker werden.

Auf das Beispiel angewendet bedeutet dies Folgendes. In den Köpfen einzelner Sprecher, die die Sequenz aus gegebenem Anlass vermehrt hören und benutzen, werden die syntagmatischen Assoziationen zwischen diesen Elementen gestärkt. Dies wird unterstützt durch die pragmatischen Assoziationen zu typischen Kontexten, in denen die Sequenz angetroffen bzw. für nützlich erachtet wird, z.B. wenn auf Missstände aufmerksam gemacht werden soll. Im Zuge der Stärkung der syntagmatischen Assoziationen werden die symbolischen und paradigmatischen Assoziationen der Teilelemente schwächer. Das heißt, dass die Bedeutungsrepräsentationen der Formen gegebenen und Anlass in der Fügung schwächer werden und damit einhergehend ihr Potenzial sinkt, paradigmatisch Kompetitoren wie Synonyme oder Antonyme zu ko-aktivieren. Im Zuge der syntagmatischen Verfestigung beginnt die ganze Sequenz allmählich, eine symbolische Assoziation zu einer holistischen Bedeutung zu gewinnen, die zunehmend von der Summe der Teilbedeutungen abweicht. Begleitend werden paradigmatische Assoziationen zu Ausdrücken mit vergleichbaren Formen und Bedeutungen (z.B. wir erinnern daran, siehe oben) und pragmatische Assoziationen zu den typischen Verwendungskontexten der ganzen Fügung gestärkt. Damit ist der Weg in Richtung Idiomatisierung in den Köpfen einzelner Sprecher eingeschlagen, ohne dass weitere Prozesse ins Spiel gebracht werden müssen. Sprecher, die die Fügung selten verwenden oder hören, durchlaufen diese kognitiven Prozesse nicht selbst. Da die Fügung bereits ein ordentliches Maß an Usualisierung erreicht hat, haben diese Sprecher aber die Möglichkeit, sich die Fügung auf dem Wege der Ko-Semiose und Ko-Adaption anzueignen. Hierfür ist erforderlich, dass sie die spezifischen semantischen und pragmatischen Eigenschaften erkennen. Bei ausreichender Usualisierung und Diffusion als holistischem Äußerungstyp erwerben immer mehr Sprecher die Fügung auf diesem Weg - also als feststehenden Ausdruck. Der erwähnte Normierungsdiskurs, der die Sequenz explizit als mehr oder weniger feststehenden Ausdruck ausweist, dürfte dazu beitragen.

Das Prinzip der syntagmatischen Verfestigung erweist sich als sehr wirkmächtige kognitive Erklärung für eine Reihe von Sprachwandelprozessen wie Lexikalisierung und Grammatikalisierung. In welcher Weise es sich entfaltet und 
wie seine Wirkungsweise von anderen Komponenten des Modells, v.a. den Konventionalisierungsprozessen, beeinflusst wird, hängt vom jeweiligen Einzelfall ab und muss durch die detaillierte Analyse der vorhandenen Daten geklärt werden. Ein rasant, d.h. in sehr kurzer Zeit ablaufender Usualisierungsprozess kann beispielsweise darauf hindeuten, dass sich eine usuelle Wortverbindung schon von Anfang an als fertiger Chunk konventionalisiert hat, statt zunächst graduell und individuell kognitiv verfestigt zu werden. Dies liegt zum Beispiel für konventionalisierte Buch- oder Filmtitel, Slogans oder auch geflügelte Wörter nahe, deren Ursprung oft mit der Verwendung assoziiert bleibt.

Sowohl graduell gefestigte als auch als fertige Chunks adaptierte usuelle Wortverbindungen können dann ihrerseits wieder als Ausgangspunkte für analogische Variantenbildungen dienen, die als Phraseoschablonen etabliert werden können (siehe den Beitrag von Katrin Steyer). Meme lassen sich hierfür als besonders schnelllebige Beispiele anführen. Wortbildungsschemata, deren Produktivitätsveränderungen im Beitrag von Martin Hilpert behandelt werden, werden ebenfalls in der Regel von einzelnen oder wenigen festen oder gefestigten Types ausgehend generalisiert. Die von Hilpert beschriebenen semantischen Gruppen, über die das englische Muster N+V-ed an Produktivität hinzugewonnen hat, lassen sich im vorliegenden Modell durch die Aktivierung paradigmatischer Assoziationen erläutern, die völlig unmotivierte Neubildungen nach dem Muster weniger wahrscheinlich machen.

Das Modell liefert für all diese verschiedenen Prozesse einen gemeinsamen Erklärungsrahmen und macht systematische Vorhersagen über syntagmatisch motivierte lexikalische Wandelprozesse, die usuelle Wortfügungen unterschiedlicher Grade an Festigkeit und Idiomatizität einerseits hervorbringen und andererseits zu neuer Produktivität verhelfen.

\section{Schlussbemerkung}

Mehr als ein grober Überblick über die Komponenten des Modells und ihre Interaktion war in diesem kurzen Beitrag nicht zu leisten. Es bleibt zu hoffen, dass das Potenzial des Modells trotz der kursorischen Darstellung erkennbar geworden ist. Es beantwortet die in Abschnitt 2 gestellten Fragen, indem es erläutert, wie sowohl die scheinbare Stabilität als auch die Dynamik des Lexikons durch die Interaktion von Sprachverwendung und individuellen kognitiven und kollektiven sozialen Prozessen unter dem Einfluss einer Reihe verschiedener Arten von Kräften hervorgebracht wird. Das Ausmaß der Stabilität und die spezifischen Manifestationen der Dynamik hängen von den zahlreichen möglichen Konstellatio- 
nen dieser Interaktion ab, die sich am besten in Anlehnung an komplex-adaptive dynamische Modelle verstehen lässt. Typische Muster der Dynamik wie Innovation, Typen des lexikalischen Wandels, Entlehnung, Festigung, Idiomatisierung, Lexikalisierung oder auch Reanalyse und Grammatikalisierung lassen sich systematisch innerhalb eines einheitlichen Modells als solche Konstellationen beschreiben.

Zum Abschluss sei noch hervorgehoben, dass das Modell so konzipiert ist, dass es einerseits im Einklang mit robusten Erkenntnissen aus der Psycholinguistik, Psychologie, Neurologie und Soziologie steht, andererseits aber auch insofern sparsam bleibt, als es sich auf eine begrenzte Zahl von Handlungen, Prozessen und Kräften beschränkt.

\section{Literatur}

Schmid, Hans-Jörg (2014): Lexico-grammatical patterns, pragmatic associations and discourse frequency. (= Constructions collocations patterns (TiLSM) 282). Berlin/New York, S. 239-293.

Schmid, Hans-Jörg (2015): A blueprint of the entrenchment-and-conventionalization model. In: Yearbook of the German Cognitive Linguistics Association 3, 1, S. 3-25.

Schmid, Hans-Jörg (2016): Why cognitive linguistics must embrace the social and pragmatic dimensions of language and how it could do so more seriously. In: Cognitive Linguistics 27, 4, S. 543-557.

Schmid, Hans-Jörg (2017a): A framework for understanding linguistic entrenchment and its psychological foundations. In: Schmid (Hg.), S. 7-36.

Schmid, Hans-Jörg (2017b): Linguistic entrenchment and its psychological foundations. In: Schmid (Hg.), S. 435-452.

Schmid, Hans-Jörg (2017) (Hg.): Entrenchment and the psychology of language learning. How we reorganize and adapt linguistic knowledge. Boston/Berlin.

Schmid, Hans-Jörg (i.Bearb.): Entrenchment and conventionalization. How usage, mind and society shape linguistic variation, change, and structure. Oxford. 